\title{
Leslie Holzhauser-Peters, Leslie True: Making Sense of Children's Thinking and Behavior: A Step by Step Tool for Understanding Children with NLD, Asperger's, HFA, PDD.NOS and Other Neurological Differences
}

\author{
Philadelphia, PA, Jessica Kingsley Publishers, 2008, \\ ISSN: 1-84310-888-7, 185 pp, \$19.95 (paper)
}

\author{
L. Jarrett Barnhill \\ Published online: 4 June 2009 \\ (C) Springer Science+Business Media, LLC 2009
}

Ms Holzhauser-Peter and True provide a practical guide to deciphering and translating cognitive, communication and behavioral aspects of children they categorize as neurologically different. This heterogeneous group includes children with nonverbal LD, Asperger's and Autistic Spectrum and other early onset neurodevelopmental disorders. The authors do not focus on specific neurological lesions or syndromes but instead on dysfunctional sensory processing, executive function and other higher cortical functions. Their strategies focus on methods of compensation for children and guidance for their target audience.

The goal of this book is to encourage parents, caregivers, teachers and clinicians to think in terms of individually designed rather program-wide interventions. Their credo is adapting social interactions and activities as well as programs to match the child's neuropsychological makeup rather than changing the child to fit the program. They offer concrete problem-solving strategy based on their assessment tool- aptly labeled systematic tool for analyzing thinking (STAT). This tool is applied to topical vignettes throughout the book with a detailed version available in the appendices.

The authors present chapters dealing with specific problems common to this population: abstract language, motor, sensory processing, spatial orientation, control/ consistency, thinking about others, social communication, flexibility, impulsivity, executive functions and anxiety. Each chapter applies the STAT format to defining and operationalizing problem areas; addressing individual temperamental and cognitive variations; formulating

L. J. Barnhill ( $₫)$

Department of Psychiatry, University of North Carolina School of Medicine, Chapel Hill, NC, USA

e-mail: Jarrett_Barnhill@med.unc.edu hypotheses then testing and modifying these to best match the child's special strengths and vulnerabilities. The authors are especially attuned to the interaction between temperament, neuropsychological deficits and challenging behaviors and adapt their interventions to match these idiosyncracies.

The STAT approach expands the assessment beyond functional behavioral analysis and standard behavioral interventions. The authors assert that functional ABA protocols tend to focus exclusively on outcome variables (reinforcing contingencies) rather than emphasizing individual patterns of misperception and idiosyncratic reactions as mitigating factors for challenging behaviors. For example, they consider the motivation for many challenging behavior to be more complicated than often specified in behavioral programs (e.g. aggression and as attention seeking or escape from demands). By taking this approach Holzhauser-Peters and True also expand the linear model of causality inherent to many behavior plans, preferring to incorporate a feedback-open systems model of biopsychosocial and environmental factors.

Although not focused on specific evidenced-based approaches this little jewel of a book provides a wealth of single case-designed, well thought out, common sense interventions. The level of analysis required to be successful also implies acquiring a great deal of information and thought about the referred individual. Perhaps this one issue is the biggest drawback. Learning the intricacies of their problem solving format might temporarily overextend already strained executive functions of many busy clinicians. But once mastered, I suspect that the time needed can be offset by program effectiveness and that the STAT can become a valuable addition to any therapeutic tool kit. 